\title{
Difference between angular momentum and pseudoangular momentum
}

\author{
Simon Streib (1) \\ Department of Physics and Astronomy, Uppsala University, Box 516, SE-75120 Uppsala, Sweden
}

(Received 29 October 2020; accepted 3 March 2021; published 16 March 2021)

\begin{abstract}
In condensed matter systems it is necessary to distinguish between the momentum of the constituents of the system and the pseudomomentum of quasiparticles. The same distinction is also valid for angular momentum and pseudoangular momentum. Based on Noether's theorem, we demonstrate that the recently discussed orbital angular momenta of phonons and magnons are pseudoangular momenta. This conceptual difference is important for a proper understanding of the transfer of angular momentum in condensed matter systems, especially in spintronics applications.
\end{abstract}

DOI: 10.1103/PhysRevB.103.L100409

In 1915, Einstein, de Haas, and Barnett demonstrated experimentally that magnetism is fundamentally related to angular momentum. When changing the magnetization of a magnet, Einstein and de Haas observed that the magnet starts to rotate, implying a transfer of angular momentum from the magnetization to the global rotation of the lattice [1], while Barnett observed the inverse effect, magnetization by rotation [2]. A few years later in 1918, Emmy Noether showed that continuous symmetries imply conservation laws [3], such as the conservation of momentum and angular momentum, which links magnetism to the most fundamental symmetries of nature.

Condensed matter systems support closely related conservation laws: the conservation of the pseudomomentum and pseudoangular momentum of quasiparticles, such as magnons and phonons. While the distinction between momentum and pseudomomentum (or quasimomentum) is well known [4-13] and is crucial for understanding the momentum of light in dielectric materials [14], the concept of pseudoangular momentum has not been widely discussed in the literature, so far only in the context of optical and acoustical vortices [15] and for chiral phonons with a discrete rotational symmetry [16-19].

The transfer of angular momentum between lattice and magnetization has been investigated in several recent works, for example, the dynamics of a single spin embedded in an elastic solid [20-22] and the transfer of angular momentum in magnetic insulators [23-27]. The spin angular momentum of phonons was first introduced by Vonsovskii and Svirskii [28], which was later rediscovered [29], as well as experimentally confirmed [30]. It has been suggested that phonon spin could

Published by the American Physical Society under the terms of the Creative Commons Attribution 4.0 International license. Further distribution of this work must maintain attribution to the author(s) and the published article's title, journal citation, and DOI. Funded by Bibsam. be used to transfer angular momentum over longer distances than would be possible with electrons or magnons [25,31,32].

Several recent publications have discussed the orbital angular momentum of phonons [21,22,33] and magnons [34,35]. The phonon orbital angular momentum plays an important role in the relaxation process of a single spin [21,22], whereas the magnon orbital angular momentum could be used for the transfer of information due to its topological stability [34] and for the manipulation of Skyrmions [35], analogous to using the orbital angular momentum of light [36]. However, there is a conceptual issue related to these recent advances: neither phonons nor magnons carry an orbital angular momentum. We demonstrate in this Letter that these orbital angular momenta are actually pseudo-orbital angular momenta, which phonons and magnons may carry.

The difference between pseudomomentum and momentum can most easily be explained by considering under which conditions they are conserved. Conservation of momentum follows from the uniformity of space and the invariance under translations of the whole system,

$$
\mathbf{r} \rightarrow \mathbf{r}+\mathbf{a},
$$

where $\mathbf{r}$ is the position coordinate and $\mathbf{a}$ is an arbitrary translation vector. The conservation of the pseudomomentum associated with a field $f(\mathbf{r})$ follows from invariance of the Lagrangian under translations of the field,

$$
f(\mathbf{r}) \rightarrow f(\mathbf{r}-\mathbf{a}),
$$

which is only valid in a uniform medium. For a crystal, pseudomomentum ("crystal momentum") is only conserved modulo $\hbar \times$ reciprocal lattice vector [37]. The pseudomomentum of a quasiparticle with wave vector $\mathbf{k}$ is simply given by $\hbar \mathbf{k}$, where $\mathbf{k}$ is defined by an expansion of the field in plane waves,

$$
f_{\mathbf{k}}(\mathbf{r}) \sim e^{i \mathbf{k} \cdot \mathbf{r}} .
$$

The difference between pseudomomentum and momentum arises only for fields in a medium and not in vacuum where both momenta are the same since the two transformations (1) and (2) are then equivalent $[6,10]$. The pseudomomentum 


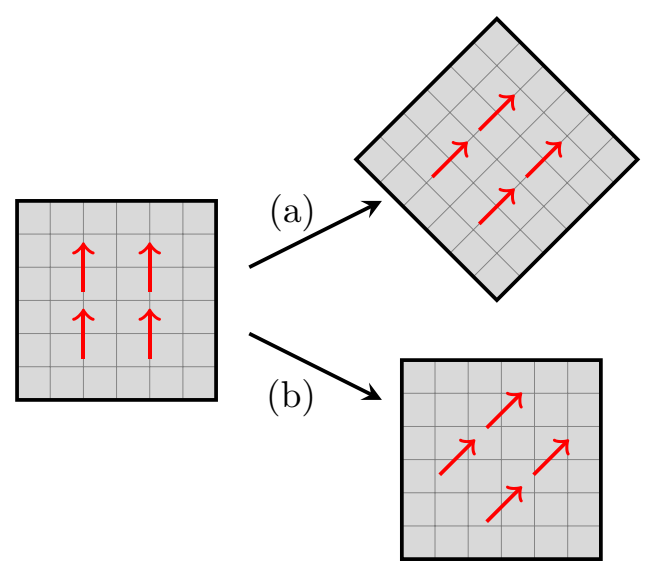

FIG. 1. (a) Invariance under rotations of the whole system implies conservation of angular momentum, while (b) invariance under rotations of fields with a fixed background implies conservation of pseudoangular momentum.

of quasiparticles in condensed matter systems is an important quantity and the qualifier "pseudo" is usually omitted in the literature. A well-known example is the momentum of phonons $[6,10,38]$. The momentum of a phonon with pseudomomentum $\hbar \mathbf{k}$ is exactly zero since the sum of the momenta of the atoms cancels. We note that it is possible to define phonons in liquids in such a way that they carry momentum $[6,7,11]$.

The definition of pseudoangular momentum is completely analogous to that of pseudomomentum. While the conservation of angular momentum follows from invariance under rotations of all the constituents of a solid, the conservation of pseudoangular momentum follows from invariance under rotations of fields while keeping the solid fixed, as illustrated in Fig. 1. The corresponding conserved quantities can then be derived from Noether's theorem [3]. Scalar fields only have an orbital angular momentum, while vector and spinor fields also have a spin angular momentum, which can be identified as the intrinsic contribution to the angular momentum that is independent of the origin of the coordinate system $[39,40]$. Similarly to pseudomomentum, pseudoangular momentum cannot be exactly conserved in a real material due to the discrete nature of atoms. However, for long-wavelength excitations the discrete atomic structure of a material is not important and the pseudoangular momentum can be approximately conserved if the material is very isotropic. A prime example is the magnetic insulator yttrium iron garnet (YIG), for which the energy dispersion of long-wavelength magnons and phonons is isotropic [41-44]. YIG is widely used in magnon spintronics applications due to its record-low magnetization damping $[32,45-47]$.

While in the case of pseudomomentum we can identify the pseudomomentum with the wave-vector $\mathbf{k}$ of a plane wave, in the case of pseudoangular momentum, we can identify the pseudoangular momentum projection along the $z$ axis with the angular momentum quantum number $m$ of an expansion of the field in terms of spherical harmonics $Y_{l m}$ for spherical geometries [22], or in terms of Bessel functions for cylindrical geometries [34]. In both cases, the fields have the typical azimuthal angle dependence

$$
f_{m}(\mathbf{r}) \sim e^{i m \phi},
$$

which is the angular analog to the plane wave (3).

Since we have these two different types of symmetries in condensed matter, invariance under translations or rotations of the whole system or only of some fields, we have to consider two different types of conservation laws: the conservation of momentum and angular momentum and the conservation of pseudomomentum and pseudoangular momentum. The elementary particles that constitute condensed matter systems are photons, electrons, and nuclei, disregarding the decomposition of nuclei into proton and neutrons or quarks, which is only relevant at much higher energy scales. For the elementary particles in vacuum their momentum and pseudomomentum and their angular momentum and pseudoangular momentum are identical, respectively.

The total angular momentum of a material can be decomposed into

$$
\mathbf{J}=\mathbf{L}_{e}+\mathbf{S}_{e}+\mathbf{L}_{n}+\mathbf{S}_{n},
$$

where $\mathbf{L}_{e}$ and $\mathbf{L}_{n}$ are the orbital angular momenta of the electrons and nuclei and $\mathbf{S}_{e}$ and $\mathbf{S}_{n}$ are their spin angular momenta. Both the orbital and spin angular momenta of the corresponding elementary fields are related to a circulating momentum flow [48-50]. In the following, we will denote the pseudomomentum and pseudoangular momentum by a tilde, e.g., $\tilde{\mathbf{P}}$ for the pseudomomentum, to distinguish them from momentum and angular momentum.

As a first example, we consider the inelastic neutron scattering process where a phonon is created [6]. The incoming neutron carries a momentum and pseudomomentum $\hbar \mathbf{k}$. In the scattering process the pseudomomentum is transferred to the phonon with pseudomomentum $\hbar \mathbf{k}$. Since the phonon does not carry momentum, the momentum $\hbar \mathbf{k}$ of the neutron has to be absorbed by the center of mass motion of the lattice. As the associated kinetic energy of a body of mass $M$ is given by $(\hbar \mathbf{k})^{2} /(2 M)$, the contribution of this kinetic energy term can be disregarded in the conservation of energy for macroscopic bodies and the energy lost by the neutron is completely transferred to the phonon. For a complete understanding of this process, we therefore have to consider the conservation of both momentum and pseudomomentum.

When considering symmetries and their corresponding conservation laws, it is important to take the boundary conditions into account. For lattice vibrations, periodic boundary conditions imply that the lattice cannot rotate and angular momentum is not conserved, as it can leave the system via the boundary conditions. Lenstra and Mandel showed that the angular momentum of the quantized electromagnetic field is also not conserved under periodic boundary conditions [51]. Experimentally, a sample is usually fixed to a sample holder, which prevents the sample from moving or rotating. Therefore, momentum and angular momentum are not conserved within the sample, whereas pseudomomentum and pseudoangular momentum can still be conserved. This has two implications: the conservation of pseudomomentum and pseudoangular momentum is immensely useful for describing experimentally relevant situations and, if we find that the total 
momentum or angular momentum is not conserved, it most likely has been lost to the boundary conditions via the lattice.

Nakane and Kohno have derived from Noether's theorem two different expression for the angular momentum of phonons [21], which they call angular momentum in field theory and angular momentum in Newtonian mechanics. Their angular momentum in field theory follows from invariance under global rotations of the phonon (or displacement) field $\mathbf{u}(\mathbf{r})$,

$$
\mathbf{u}(\mathbf{r}) \rightarrow \mathcal{R} \mathbf{u}\left(\mathcal{R}^{-1} \mathbf{r}\right),
$$

where the rotation matrix $\mathcal{R}$ appears twice because both the positions and the directions of the fields are rotated. The phonon field $\mathbf{u}(\mathbf{r})$ is a vector field that describes the displacement of the atoms at position $\mathbf{r}$. From our discussion of pseudoangular momentum, it is clear that this angular momentum corresponds to the pseudoangular momentum of the phonon field, since it is based on the invariance under rotations of an emergent field. It takes the form [21]

$$
\begin{gathered}
\tilde{\mathbf{J}}_{\mathrm{ph}}=\tilde{\mathbf{L}}_{\mathrm{ph}}+\tilde{\mathbf{S}}_{\mathrm{ph}} \\
\tilde{\mathbf{L}}_{\mathrm{ph}}=\sum_{\alpha=x, y, z} \int \rho \dot{u}_{\alpha} \mathbf{r} \times(-\nabla) u_{\alpha} d^{3} r \\
\tilde{\mathbf{S}}_{\mathrm{ph}}=\int \rho(\mathbf{u} \times \dot{\mathbf{u}}) d^{3} r
\end{gathered}
$$

where $\rho$ is the mass density of the solid and both the orbital contribution $\tilde{\mathbf{L}}_{\mathrm{ph}}$ and the spin contribution $\tilde{\mathbf{S}}_{\mathrm{ph}}$ are bilinear in the phonon field. The corresponding phonon pseudomomentum reads [21]

$$
\tilde{\mathbf{P}}_{\mathrm{ph}}=\sum_{\alpha=x, y, z} \int \rho \dot{u}_{\alpha}(-\nabla) u_{\alpha} d^{3} r .
$$

The "Newtonian" angular momentum derives from invariance under rotations of the whole lattice [21],

$$
\mathbf{u}(\mathbf{r})+\mathbf{r} \rightarrow \mathcal{R}[\mathbf{u}(\mathbf{r})+\mathbf{r}] .
$$

Therefore this angular momentum is the orbital angular momentum of the atoms and is given by [20,21]

$$
\begin{gathered}
\mathbf{J}_{\mathrm{ph}}=\mathbf{L}_{\mathrm{ph}}+\mathbf{S}_{\mathrm{ph}}=\int \rho([\mathbf{u}+\mathbf{r}] \times \dot{\mathbf{u}}) d^{3} r, \\
\mathbf{L}_{\mathrm{ph}}=\int \rho(\mathbf{r} \times \dot{\mathbf{u}}) d^{3} r, \\
\mathbf{S}_{\mathrm{ph}}=\int \rho(\mathbf{u} \times \dot{\mathbf{u}}) d^{3} r,
\end{gathered}
$$

where the spin contribution $\mathbf{S}_{\mathrm{ph}}$ is formally identified as the intrinsic contribution which is independent of the origin of the coordinate system [20]. The momentum of the lattice,

$$
\mathbf{P}_{\mathrm{ph}}=\int \rho \dot{\mathbf{u}} d^{3} r,
$$

is only finite when there is a center of mass motion of the whole lattice, contrary to the pseudomomentum which is finite for phonon excitations without a center of mass motion.

Comparing the angular momentum and pseudoangular momentum associated with the phonon field, we see that spin and pseudospin are identical, while orbital and pseudo-orbital angular momentum are not. Both the momentum $\mathbf{P}_{\mathrm{ph}}$ and the orbital angular momentum $\mathbf{L}_{\mathrm{ph}}$ are linear in the phonon field and vanish for phonon excitations, e.g., for any plane wave with $\mathbf{k} \neq 0$,

$$
\mathbf{P}_{\mathrm{ph}} \sim \int e^{i \mathbf{k} \cdot \mathbf{r}} d^{3} r=0 .
$$

A finite value of $\mathbf{L}_{\mathrm{ph}}$ corresponds to a global rotation of the whole lattice. Like $\mathbf{P}_{\mathrm{ph}}$ and $\tilde{\mathbf{P}}_{\mathrm{ph}}, \mathbf{L}_{\mathrm{ph}}$ and $\tilde{\mathbf{L}}_{\mathrm{ph}}$ do not represent the same physical quantity, contrary to the suggestion in Ref. [21]. The phonon spin is finite for circularly polarized phonons, analogous to the spin angular momentum of light [52].

The importance of distinguishing between the angular momentum and the pseudoangular momentum of phonons can be demonstrated by considering two recent publications. Reference [22] considers a single spin embedded in an elastic medium, where angular momentum is transferred from the spin to the orbital angular momentum of phonons. Since we have established that phonons do not carry orbital angular momentum, the phonon orbital angular momentum in Ref. [22] has to be the pseudo-orbital angular momentum $\tilde{\mathbf{L}}_{\mathrm{ph}}$, which phonons may carry. This can be confirmed by considering that the orbital angular momentum of the phonon modes in Ref. [22] is based on an expansion in spherical harmonics $Y_{l m}$, which is analogous to an expansion in plane waves with pseudomomentum $\mathbf{k}$. The angular momentum of the embedded spin enters the conversation of pseudoangular momentum because the system is only invariant under rotations of both the phonon fields and the spin direction as both are coupled [21]. Therefore a change in spin has to be balanced by a change of the phonon pseudoangular momentum. The key question is: what happens to the angular momentum of the spin if it is not transferred to the phonons? As Ref. [22] applies periodic boundary conditions to the phonons, the total angular momentum is not conserved. Physically, the angular momentum is in this case absorbed by the boundary conditions and would be transferred to a global rotation of the lattice if it were not for the boundary conditions that prevent such a rotation. Reference [24], on the other hand, considers the transfer of angular momentum among magnons, phonons, and the global lattice rotation of a magnetic insulator. There, the orbital angular momentum of phonons is not mentioned at all since the quantity under consideration is the angular momentum of the phonons, which is carried only by the phonon spin. The apparent contradiction between Refs. [22] and [24] is resolved by taking into account that Ref. [22] is considering the pseudoangular momentum and Ref. [24] the angular momentum of phonons. Both approaches are valid, but it is important to be aware of which kind of angular momentum is described and which conservation laws apply.

Similarly to the phonon case, we analyze next the angular momentum of magnons in a collinear ferromagnet, motivated by recent advances related to their orbital angular momentum [34,35]. We define the classical magnon field $\psi(\mathbf{r})$ in the following way. We start from the Holstein-Primakoff 
transformation of the spin operator $\hat{S}_{i}^{ \pm}=\hat{S}_{i}^{x} \pm i \hat{S}_{i}^{y}$ at lattice site $i[53]$,

$$
\begin{gathered}
\hat{S}_{i}^{+}=\hbar \sqrt{2 S} \sqrt{1-\frac{\hat{n}_{i}}{2 S}} \hat{b}_{i}, \\
\hat{S}_{i}^{-}=\hbar \sqrt{2 S} \hat{b}_{i}^{\dagger} \sqrt{1-\frac{\hat{n}_{i}}{2 S}}, \\
\hat{S}_{i}^{z}=\hbar\left(S-\hat{n}_{i}\right),
\end{gathered}
$$

where $S$ is the dimensionless spin quantum number, $\hat{n}_{i}=\hat{b}_{i}^{\dagger} \hat{b}_{i}$ is the magnon number operator, and $\hat{b}_{i}^{\dagger}$ and $\hat{b}_{i}$ are the magnon creation and annihilation operators with the commutation relations

$$
\left[\hat{b}_{i}, \hat{b}_{j}^{\dagger}\right]=\delta_{i j}, \quad\left[\hat{b}_{i}, \hat{b}_{j}\right]=\left[\hat{b}_{i}^{\dagger}, \hat{b}_{j}^{\dagger}\right]=0 .
$$

We can introduce the continuous magnon field $\hat{\psi}(\mathbf{r})$,

$$
\hat{b}_{i}=\frac{1}{\sqrt{V_{i}}} \int_{V_{i}} \hat{\psi}(\mathbf{r}) d^{3} r
$$

where $V_{i}$ is the volume of the unit cell associated with the spin at lattice site $i$. The replacement of the magnon operators $\hat{b}_{i}$ by the field $\hat{\psi}(\mathbf{r})$ is valid for long-wavelength magnons (i.e., in the continuum limit). The normalization is chosen such that the magnon field fulfills

$$
\left[\hat{\psi}(\mathbf{r}), \hat{\psi}^{\dagger}\left(\mathbf{r}^{\prime}\right)\right]=\delta\left(\mathbf{r}-\mathbf{r}^{\prime}\right) .
$$

This allows us to define the canonically conjugate field

$$
\hat{\pi}(\mathbf{r})=i \hbar \hat{\psi}^{\dagger}(\mathbf{r})
$$

with the canonical commutation relation

$$
\left[\hat{\psi}(\mathbf{r}), \hat{\pi}\left(\mathbf{r}^{\prime}\right)\right]=i \hbar \delta\left(\mathbf{r}-\mathbf{r}^{\prime}\right) .
$$

The corresponding classical magnon field fulfills then the canonical Poisson bracket relations at equal times,

$$
\left\{\psi(\mathbf{r}, t), \pi\left(\mathbf{r}^{\prime}, t\right)\right\}=\delta\left(\mathbf{r}-\mathbf{r}^{\prime}\right) .
$$

Because a general spin Hamiltonian $\mathcal{H}[\psi, \pi]$ is a functional of the fields and their gradients but not of their time derivatives, we obtain the classical Lagrangian [40]

$$
\mathcal{L}=\int \pi \dot{\psi} d^{3} r-\mathcal{H}[\psi, \pi] .
$$

It is now straightforward to apply Noether's theorem to this Lagrangian [40] and to derive the classical magnon pseudomomentum and pseudoangular momentum,

$$
\begin{gathered}
\tilde{\mathbf{P}}_{\mathrm{m}}=-i \hbar \int \psi^{*} \nabla \psi d^{3} r, \\
\tilde{\mathbf{L}}_{\mathrm{m}}=-i \hbar \int \mathbf{r} \times\left(\psi^{*} \nabla \psi\right) d^{3} r .
\end{gathered}
$$

The magnon field $\psi(\mathbf{r})$ is a complex scalar field and therefore the magnon pseudoangular momentum has only an orbital contribution and no pseudospin. The pseudomomentum $\hbar \mathbf{k}$ of magnons enters the conservation of pseudomomentum, which is, for example, important in spin transfer processes [54,55]. The total spin angular momentum is determined by the number of excited magnons,

$$
\begin{aligned}
S_{\mathrm{tot}}^{z} & =S_{\mathrm{tot}}^{0}-\hbar \sum_{i}\left\langle\hat{n}_{i}\right\rangle \\
& =S_{\mathrm{tot}}^{0}-\hbar \int \psi^{*}(\mathbf{r}) \psi(\mathbf{r}) d^{3} r,
\end{aligned}
$$

where $S_{\mathrm{tot}}^{0}=\hbar N S$, with $N$ the number of lattice sites, is the total spin if no magnons are excited.

We note that it is also possible to derive the pseudoangular momentum of the magnetization field $\mathbf{M}(\mathbf{r})$, which is a vector field with a spin contribution to its pseudoangular momentum [56,57], although there are complications with respect to the application of Noether's theorem since the resulting pseudomomenta are gauge dependent and not uniquely defined [57-60]. This is, however, not a fundamental issue because these quantities are pseudomomenta and not proper momenta, which would have to be well defined. Similarly, the pseudomomentum and pseudoangular momentum of magnons are only defined with respect to a given magnon quantization axis, which is in principle arbitrary. However, magnons usually describe fluctuations with respect to a given magnetic equilibrium configuration that physically defines a preferred quantization axis for each spin.

By comparing our results for the magnon pseudomomentum and pseudoangular momentum [Eqs. (27) and (28)] with the magnon momentum and magnon orbital angular momentum of Ref. [34], we find that they are indeed the same quantities: the pseudomomentum and pseudoangular momentum of magnons. Reference [35] uses instead the definition of the pseudo-orbital angular momentum of the magnetization field [56,57].

Since a magnon excitation is only associated with a change of the magnetization and therefore of the angular momentum, a magnon cannot have any momentum, only pseudomomentum. The angular momentum associated with the magnon field is determined by the number of magnons alone [Eq. (29)] and does not depend on the spatial structure of the magnon field, whereas the pseudo-orbital angular momentum $\tilde{\mathbf{L}}_{\mathrm{m}}$ shows such a dependence and does not correspond to any contribution to the angular momentum. If the magnetization is not due to the electron spin alone but does also have an orbital contribution, then there is a correlation between the electron orbital angular momentum and the number of magnons.

We point out that the recently proposed orbital magnetic moments of phonons [61] and magnons [62] are not related to pseudoangular momentum. Magnetic moments are always due to the angular momentum of charged particles. In the case of Ref. [61], the underlying angular momentum is the orbital angular momentum of ions and in the case of Ref. [62] the orbital angular momentum of electrons.

In summary, we have shown that the conservation of pseudoangular momentum arises when a condensed matter system is invariant under rotations of the emergent fields that are associated with quasiparticles. We have considered the pseudoangular momentum of phonons and magnons, which are derived from Noether's theorem, and demonstrated that 
the recently discussed orbital angular momenta of phonons $[21,22,33]$ and magnons [34,35] are in fact pseudoangular momenta. In general, we conclude that when Noether's theorem is applied to emergent quasiparticle fields, the resulting conserved momenta are pseudomomenta. The examples discussed here show that it is important to consider the difference between angular momentum and pseudoangular momentum, which is especially important in spintronics applications where angular momentum is used as an information carrier. While angular momentum is in principle always conserved in a closed system, pseudoangular momentum is not exactly conserved in real materials, which should be kept in mind for potential experimental applications. The distinction between angular momentum and pseudoangular momentum could also be crucial for the analysis of the angular momentum associated with the pseudospin in graphene, which has been argued to represent a real angular momentum $[63,64]$.

I thank Charles Sebens for discussions on angular momentum and spin, Mikhael Katsnelson and Johan Mentink for discussing the concept of pseudomomentum, and Jorge E. Hirsch for discussions on the transfer of angular momentum in superconductors and ferromagnets and his hospitality after the cancellation of the APS March Meeting 2020. Special thanks go to Anna Delin, Danny Thonig, and Olle Eriksson for feedback on this research project. Financial support from the Knut and Alice Wallenberg Foundation through Grant No. 2018.0060 is gratefully acknowledged.
[1] A. Einstein and W. J. de Haas, Experimental Proof of the Existence of Ampère's Molecular currents, Proc. KNAW 18, 696 (1915).

[2] S. J. Barnett, Magnetization by rotation, Phys. Rev. 6, 239 (1915).

[3] E. Noether, Invariante Variationsprobleme, Nachr. Ges. Wiss. Goettingen, Math. Phys. Kl. 2, 235 (1918).

[4] J. P. Gordon, Radiation forces and momenta in dielectric media, Phys. Rev. A 8, 14 (1973).

[5] R. Peierls, The momentum of light in a refracting medium, Proc. R. Soc. London A 347, 475 (1976).

[6] R. Peierls, Surprises in Theoretical Physics (Princeton University Press, Princeton, 1979).

[7] A. Thellung, Two-fluid equations for phonons without momentum, Ann. Phys. (NY) 127, 289 (1980).

[8] M. E. Mcintyre, On the 'wave momentum' myth, J. Fluid Mech. 106, 331 (1981).

[9] D. F. Nelson, Momentum, pseudomomentum, and wave momentum: Toward resolving the Minkowski-Abraham controversy, Phys. Rev. A 44, 3985 (1991).

[10] R. Peierls, More Surprises in Theoretical Physics (Princeton University Press, Princeton, 1991).

[11] A. Thellung, Momentum and Quasimomentum in the Physics of Condensed Matter, in Die Kunst of Phonons: Lectures from the Winter School of Theoretical Physics (Springer US, Boston, MA, 1994).

[12] M. Stone, Phonons and forces: Momentum versus pseudomomentum in moving fluids, in Artificial Black Holes (World Scientific, Singapore, 2002).

[13] E. J. Heller and D. Kim, Schrödinger Correspondence Applied to Crystals, J. Phys. Chem. A 123, 4379 (2019).

[14] R. N. C. Pfeifer, T. A. Nieminen, N. R. Heckenberg, and H. Rubinsztein-Dunlop, Colloquium: Momentum of an electromagnetic wave in dielectric media, Rev. Mod. Phys. 79, 1197 (2007).

[15] J.-L. Thomas and R. Marchiano, Pseudo Angular Momentum and Topological Charge Conservation for Nonlinear Acoustical Vortices, Phys. Rev. Lett. 91, 244302 (2003).

[16] L. Zhang and Q. Niu, Chiral Phonons at High-Symmetry Points in Monolayer Hexagonal Lattices, Phys. Rev. Lett. 115, 115502 (2015).
[17] H. Zhu, J. Yi, M.-Y. Li, J. Xiao, L. Zhang, C.-W. Yang, R. A. Kaindl, L.-J. Li, Y. Wang, and X. Zhang, Observation of chiral phonons, Science 359, 579 (2018).

[18] Y. Tatsumi, T. Kaneko, and R. Saito, Conservation law of angular momentum in helicity-dependent Raman and Rayleigh scattering, Phys. Rev. B 97, 195444 (2018).

[19] W. Zhang, A. Srivastava, X. Li, and L. Zhang, Chiral phonons in the indirect optical transition of a $\mathrm{MoS}_{2} / \mathrm{WS}_{2}$ heterostructure, Phys. Rev. B 102, 174301 (2020).

[20] D. A. Garanin and E. M. Chudnovsky, Angular momentum in spin-phonon processes, Phys. Rev. B 92, 024421 (2015).

[21] J. J. Nakane and H. Kohno, Angular momentum of phonons and its application to single-spin relaxation, Phys. Rev. B 97, 174403 (2018).

[22] J. H. Mentink, M. I. Katsnelson, and M. Lemeshko, Quantum many-body dynamics of the Einstein-de Haas effect, Phys. Rev. B 99, 064428 (2019).

[23] S. Streib, H. Keshtgar, and G. E. W. Bauer, Damping of Magnetization Dynamics by Phonon Pumping, Phys. Rev. Lett. 121, 027202 (2018).

[24] A. Rückriegel, S. Streib, G. E. W. Bauer, and R. A. Duine, Angular momentum conservation and phonon spin in magnetic insulators, Phys. Rev. B 101, 104402 (2020).

[25] A. Rückriegel and R. A. Duine, Long-Range Phonon Spin Transport in Ferromagnet-Nonmagnetic Insulator Heterostructures, Phys. Rev. Lett. 124, 117201 (2020).

[26] X. Zhang, G. E. W. Bauer, and T. Yu, Unidirectional Pumping of Phonons by Magnetization Dynamics, Phys. Rev. Lett. 125, 077203 (2020).

[27] D. M. Juraschek, P. Narang, and N. A. Spaldin, Phonomagnetic analogs to opto-magnetic effects, Phys. Rev. Research 2, 043035 (2020).

[28] S. V. Vonsovskii and M. S. Svirskii, Phonon Spin, Sov. Phys. Solid State 3, 1568 (1962).

[29] L. Zhang and Q. Niu, Angular Momentum of Phonons and the Einstein-de Haas Effect, Phys. Rev. Lett. 112, 085503 (2014).

[30] J. Holanda, D. S. Maior, A. Azevedo, and S. M. Rezende, Detecting the phonon spin in magnon-phonon conversion experiments, Nat. Phys. 14, 500 (2018).

[31] K. An, A. N. Litvinenko, R. Kohno, A. A. Fuad, V. V. Naletov, L. Vila, U. Ebels, G. de Loubens, H. Hurdequint, N. Beaulieu, 
J. Ben Youssef, N. Vukadinovic, G. E. W. Bauer, A. N. Slavin, V. S. Tiberkevich, and O. Klein, Coherent long-range transfer of angular momentum between magnon kittel modes by phonons, Phys. Rev. B 101, 060407(R) (2020).

[32] A. Brataas, B. van Wees, O. Klein, G. de Loubens, and M. Viret, Spin insulatronics, Phys. Rep. 885, 1 (2020).

[33] M. K. Ayub, S. Ali, and J. T. Mendonca, Phonons with orbital angular momentum, Phys. Plasmas 18, 102117 (2011).

[34] C. Jia, D. Ma, A. F. Schäffer, and J. Berakdar, Twisted magnon beams carrying orbital angular momentum, Nat. Commun. 10, 2077 (2019).

[35] Y. Jiang, H. Y. Yuan, Z.-X. Li, Z. Wang, H. W. Zhang, Y. Cao, and P. Yan, Twisted Magnon as a Magnetic Tweezer, Phys. Rev. Lett. 124, 217204 (2020).

[36] W. Yang, H. Yang, Y. Cao, and P. Yan, Photonic orbital angular momentum transfer and magnetic skyrmion rotation, Opt. Express 26, 8778 (2018).

[37] N. Ashcroft and N. Mermin, Solid State Physics (Saunders College Publishing, Philadelphia, 1976).

[38] C. Kittel, Introduction to Solid State Physics, 7th ed. (Wiley, New York, 1996).

[39] D. E. Soper, Classical Field Theory (Wiley, New York, 1976).

[40] W. Greiner and J. Reinhardt, Field Quantization (Springer, Berlin, 1996).

[41] V. Cherepanov, I. Kolokolov, and V. L'vov, The saga of YIG: Spectra, thermodynamics, interaction and relaxation of magnons in a complex magnet, Phys. Rep. 229, 81 (1993).

[42] A. G. Gurevich and G. A. Melkov, Magnetization Oscillations and Waves (CRC, Boca Raton, FL, 1996).

[43] S. Maehrlein, Nonlinear terahertz phononics: A novel route to controlling matter, Ph.D. thesis, Freie Universität Berlin, 2017.

[44] S. F. Maehrlein, I. Radu, P. Maldonado, A. Paarmann, M. Gensch, A. M. Kalashnikova, R. V. Pisarev, M. Wolf, P. M. Oppeneer, J. Barker, and T. Kampfrath, Dissecting spin-phonon equilibration in ferrimagnetic insulators by ultrafast lattice excitation, Sci. Adv. 4, eaar5164 (2018).

[45] V. V. Kruglyak, S. O. Demokritov, and D. Grundler, Magnonics, J. Phys. D: Appl. Phys. 43, 264001 (2010).

[46] A. V. Chumak, V. I. Vasyuchka, A. A. Serga, and B. Hillebrands, Magnon spintronics, Nat. Phys. 11, 453 (2015).

[47] S. A. Nikitov, D. V. Kalyabin, I. V. Lisenkov, A. Slavin, Y. N. Barabanenkov, S. A. Osokin, A. V. Sadovnikov, E. N. Beginin, M. A. Morozova, Y. A. Filimonov, Y. V. Khivintsev, S. L. Vysotsky, V. K. Sakharov, and E. S. Pavlov, Magnonics: A new research area in spintronics and spin wave electronics, Phys. Usp. 58, 1002 (2015).

[48] H. C. Ohanian, What is spin? Am. J. Phys. 54, 500 (1986).

[49] C. T. Sebens, How electrons spin, Stud. Hist. Philos. Sci. B 68, 40 (2019).

[50] K. Y. Bliokh, H. Punzmann, H. Xia, F. Nori, and M. Shats, Relativistic field-theory spin and momentum in water waves (2020), arXiv:2009.03245.

[51] D. Lenstra and L. Mandel, Angular momentum of the quantized electromagnetic field with periodic boundary conditions, Phys. Rev. A 26, 3428 (1982).

[52] S. J. van Enk and G. Nienhuis, Spin and Orbital Angular Momentum of Photons, Europhys. Lett. 25, 497 (1994).

[53] T. Holstein and H. Primakoff, Field dependence of the intrinsic domain magnetization of a ferromagnet, Phys. Rev. 58, 1098 (1940).

[54] A. Mitrofanov and S. Urazhdin, Energy and momentum conservation in spin transfer, Phys. Rev. B 102, 184402 (2020).

[55] N. Tramsen, A. Mitrofanov, and S. Urazhdin, Effects of the dynamical magnetization state on spin transfer (2021), arXiv:2101.08868.

[56] E. V. Gol'dshtein and V. M. Tsukernik, Angular momentum of a Heisenberg ferromagnet with a magnetic dipole interaction, Sov. Phys. JETP 60, 764 (1984).

[57] P. Yan, A. Kamra, Y. Cao, and G. E. W. Bauer, Angular and linear momentum of excited ferromagnets, Phys. Rev. B 88, 144413 (2013).

[58] N. Papanicolaou and T. Tomaras, Dynamics of magnetic vortices, Nucl. Phys. B 360, 425 (1991).

[59] O. Tchernyshyov, Conserved momenta of a ferromagnetic soliton, Ann. Phys. (NY) 363, 98 (2015).

[60] S. Dasgupta and O. Tchernyshyov, Energy-momentum tensor of a ferromagnet, Phys. Rev. B 98, 224401 (2018).

[61] D. M. Juraschek and N. A. Spaldin, Orbital magnetic moments of phonons, Phys. Rev. Materials 3, 064405 (2019).

[62] R. R. Neumann, A. Mook, J. Henk, and I. Mertig, Orbital Magnetic Moment of Magnons, Phys. Rev. Lett. 125, 117209 (2020).

[63] M. Mecklenburg and B. C. Regan, Spin and the Honeycomb Lattice: Lessons from Graphene, Phys. Rev. Lett. 106, 116803 (2011).

[64] D. Song, V. Paltoglou, S. Liu, Y. Zhu, D. Gallardo, L. Tang, J. Xu, M. Ablowitz, N. K. Efremidis, and Z. Chen, Unveiling pseudospin and angular momentum in photonic graphene, Nat. Commun. 6, 6272 (2015). 